\title{
A Platform for the Agent-based Control of HVAC Systems
}

\author{
Roozbeh Sangi Felix Bünning Johannes Fütterer Dirk Müller \\ Institute for Energy Efficient Buildings and Indoor Climate, E.ON Energy Research Center, RWTH Aachen \\ University, Germany, rsangi@eonerc.rwth-aachen.de
}

\begin{abstract}
Attempts to develop efficient and environmentally friendly building energy systems have led to modern complex energy concepts for buildings, which have consequently initiated a need for new control strategies for them. Multiagent control, which is known with other names like agent-based control, offers a promising solution to this challenge. To the knowledge of the authors, there are 96 platforms for multi-agent systems in different programming languages available, which are mostly java-based and mainly used in logistic applications, but there is no platform in the modeling language Modelica, which is widely used for simulation of dynamic systems, especially buildings performance simulation. This lack motivated the authors to develop a platform for agent-based control of HAVC systems. The platform eliminates the dependency of models developed in Modelica on an extra interface, which is usually required to couple the models to the platforms written in any programming languages other than Modelica. This paper presents the structure of the platform and explains how the agents' communications work. The flexibility of the optimization objective is ensured through the definition of readily interchangeable cost functions. The applicability and functionality of the platform are proved by applying the platform in the control of building energy systems examples.

Keywords: Agent-based control, Building energy systems, Control, HVAC, Modelica, Multi-Agent System
\end{abstract}

\section{Introduction}

The amount of energy used for heating and cooling in the building sector is about one third of the total energy consumed in the world. The finiteness of natural energy resources on the one hand, and the ever-increasing demand for energy in the world on the other hand, necessitate the development of systematic approaches for improving the efficiency of building energy systems as well as minimizing the usage of primary energy resources and the damaging impacts and harmful effects on the environment (Sangi et al., 2014). Taking into account that renewable energy sources for the building sector such as photovoltaics, heat pumps and combined-heat-and-power units are becoming profitable, such components are installed in private and commercial buildings with increasing quantity. Often more than one of such components are operated in parallel to increase cost effectiveness and the security of supply.

Consequently, the complexity of building energy systems has severely increased in the recent past. Therewith the need for controlling concepts that can handle such complexity has arisen. Besides concepts like ModelPredictive control and Artificial Neural Networks (Afram and Janabi-Sharifi, 2014), the concept of agent-based control realized through Multi-Agent Systems (MAS) promises good results in the area of HVAC control (Huber et al., 2015).

Multi-Agent Systems were successfully applied in the areas of logistics and telecommunication in the past (Verein Deutscher Ingenieure, 2010). Inherently this concept is suited to solve complex control problems and is therefore predestined for the control of complex energy systems. For the development of such systems, tools for multiple programming languages and programming environments are available (Allan, 2010), but not for the object-oriented language Modelica.

Modelica is a modelling language commonly used for the dynamic simulation of thermo-hydraulic systems. It is receiving growing attention in the use of modeling and simulation of building energy systems, as recent studies indicate: In (Wetter et al., 2014) a Modelica library for the simulation of building energy systems is introduced. (Ali et al., 2013), (Perera et al., 2016), (Sangi et al., 2016) and (Fuchs et al., 2016) use Modelica in order to model, simulate and investigate in building energy systems as well as district heating systems. MAS will play an important role in the control of future building energy systems (see 2.2). Consequently, a Modelica library for MAS will be needed.

In the course of this work a library for the agent-based control of building energy systems in the modeling language Modelica is developed, implemented and finally validated in a case study. The library allows "plug-andplay" implementation of MAS into any model of a building energy system in the Modelica environment, thus allowing the investigation in agent-based building energy system control through dynamic simulation. It depicts a solution that through UDP/IP-communication can be run on distributed machines, enabling the user to integrate both software and hardware into the optimization problem. Furthermore, the cost functions can be changed without interfering with the agent system leading to a flexible solution in which the individual user can optimize their energy system for an individual optimization goal with only 
minor engineering effort.

In the following an overview on multi-agent system is presented and the system structure is described. The developed library for agent-based control is also introduced following by an example that demonstrates the application of the library in building energy systems.

\section{Overview on Multi-Agent Systems}

\subsection{The Concept of Agents and Multi-Agent Systems}

Agents The concept of agent-based control is a concept which allows to control complex systems by splitting the main objective of the system into smaller objectives which so-called agents try to obtain by interacting with each other. Although the concept is widely spread in the scientific area, especially in the field of computer science and information technologies, there is no unified definition of the term agent.

After the term first appeared in the context of a dissertation in 1985, in which the term agent is connected with the attributes of autonomy and problem-solving behaviour (Rosenschein, 1985), further attributes such as proactivity and the ability to work towards higher goals (Wernstedt, 2005), the ability to perceive the changes of their surroundings and to react on them (Divenyi, 2013), the ability of rational calculation and organization of actions to achieve higher aims as well as permanent activeness (Kirn, 2002), socialness and truthfulness (Bellifemine et al., 2007) were defined by various authors.

In VDI 2653 agents are defined as encapsulated entities, hardware or software, with specified objectives. An agent attempts to achieve these objectives through its autonomous behaviour, in interacting with other agents and their surrounding. In addition, several characteristics such as autonomy, scope of action, interaction, encapsulation, persistence, goal-orientation and reactivity are defined.

Multi-Agent Systems VDI 2653 describes Multi-Agent Systems (MAS) as a set of agents interacting to fulfil one or more tasks. Bellifemine describes MAS as entities that can model complex systems and introduces the possibility of agents having common or conflicting goals. These agents are able to interact with each other both indirectly, by acting on the environment, or directly via communication and negotiation. Depending on their task they may cooperate to reach a common goal or compete to achieve their own aims. (Bellifemine et al., 2007)

An MAS can be used to control complex systems. One advantage over a holistic control concept is the possibility of splitting the often very complex control problem into sub-problems and -tasks and dividing them between the agents. This approach is beneficial for the developer as the analysis of those sub-problems is more accessible than the analysis of the holistic problem and thus also the implementation of the systems solving these problems. Furthermore, an agent-based approach has the advantage of being more easily adjustable during the runtime of the system as new agents can be implemented and added to the system.

\subsection{Use of Multi-Agent Systems in energy- system control}

MAS have received growing recognition in various fields over the past few years. Beginning in the fields of computer science, such as Human Computer Interaction, where agents help the user depending on their already existing experience with the software, or Information Retrieval, where agents search the Internet for specific information for their user, now agent-based systems have also reached the field of logistics and telecommunication (Verein Deutscher Ingenieure, 2010). As a consequence of growing complexity in the various fields of science, MAS also receive growing attention in the fields of chemistry, biology, physics, sociology and economics (Kirn, 2002). In recent years the field of energy generation and distribution has become much more complex due to the increase of renewable energies and the concept of smartand micro-grids. MAS depict a promising technology to control the described energy systems.

Regarding the use of MAS to control classical smartand micro-grids, i.e. systems which generate and distribute electricity, a lot of research has been conducted (for example (Jiang, 2006), (Kok et al., 2012), (Kuznetsova et al., 2014), (Ye et al., 2015), (Karavas et al., 2015), (Khan et al., 2016), (Radhakrishnan and Srinivasan, 2016), (Rahman et al., 2016), (Xydas et al., 2016), (Ansari et al., 2016)). However, also the use of MAS for complex energy systems for the generation and distribution of heat or cold, such as building energy systems, HVAC systems and district heating grids, has recently gained growing attention.

In (Huberman and Clearwater, 1995) a market-based MAS is used to distribute warm and cold air in an office building. The system uses a double-blind auction procedure in which agents bid to buy and sell warm and cold air. The auction is managed by a central auctioneer. Experiments with a real office building show an even temperature distribution in the building without leading to excessive actuator movement.

(Qiao et al., 2006) introduces an MAS which combines the control of a building energy system with user interaction. The system is built of personal agents, local agents and central agents. Personal agents act as teachable assistants which carry personal user information, such as preferred room temperature, humidity and the current location of the user. Local agents act as mediators, policy enforcers and information providers. The tasks of the central agent are decision aggregation and interfacing services.

A similar system based on personal agents, local agents and central agents is used in (Yang and Wang, 2013). Personal agents are developed to predict user preferences by learning their behaviours. Local agents act as mediators, information providers, decision makers and control executors while the central agent facilitates collaboration be- 
tween the local agents while regarding the overall system goal. The functionality of the system regarding effective control of the building energy system while satisfying occupants' demands is proven with simulations and case studies.

In (Wang et al., 2011) a system using only central and local agents is used. The central agent contains the main intelligence of the system. It calculates set point of temperature, illumination and humidity based on user preferences and outdoor information using particle swarm optimization. The local agents use fuzzy controllers to control the actuators in order to reach said set points.

In (Wang et al., 2012) an indoor energy and comfort management system based on information fusion and a multi-agent control system is proposed. A multi-agent building control system with particle swarm optimization is built to achieve the smart building control goal, which is to maximize the comfort index using minimum power consumption.

(Davidsson and Boman, 2000) uses an MAS consisting of personal comfort agents, room agents, environmental parameter agents and badge system agents to control temperature and illumination in a building. Following simulation results, the system is able to reduce energy use while maintaining user satisfaction.

In (Mokhtar et al., 2013) an already existing MAS for building heat distribution control is updated with an ARTMAP, a type of artificial neural network with learning capabilities. Simulation results show the proposed intelligent MAS is able to maximize the use of a ground source heat pump effectively by profiling, predicting and coordinating its usage with other energy resources.

(Mokhtar et al., 2014) uses a similar MAS based on ARTMAP to control a building energy system based on learned user preferences. Simulation results show that the system provides better energy control and thermal comfort management than a reference rule-based MAS.

In (Hurtado et al., 2015) an agent-based approach to optimize the interaction of smart-grids and building energy systems is developed. Particle swarm optimization is used to maximize comfort and energy efficiency. It is shown that the operation of the building energy system with the MAS allows the support of the voltage control in the smart-grid.

(van Pruissen et al., 2014) presents a solution based on electronic market principles called HeatMatcher. HeatMatcher is a P2P system based on PowerMatcher (Kok et al., 2012), which is a general purpose coordination mechanism for balancing supply and demand in electricity networks. The system features trading of heat on two different time scales depending on the inertia of the components involved in trading. The MAS is tested with a floor heating system connected to a heat pump and a boiler and shows more energy efficient operation than a reference centralized controller.

In (Huber et al., 2015) an MAS based on consumer agents and supply agents is introduced. Consumer agents recognize heating or cooling demands and request them from supply agents. Supply agents estimate the corresponding costs. The control of the energy system is governed by negotiation between those agents. The system is tested with a hardware-in-the-loop test bench consisting of a central air handling unit and four rooms. Results show basic functionality of the system.

The developed MAS within the course of this research offers a flexible approach to the control of building-energy systems. The MAS can, without any adaptation necessary, be applied to any building energy system in the simulation environment of Modelica. With only minor effort it can also be used to control any energy systems, e.g. electrical grids or district heating networks. Furthermore, the MAS can also be used for real life applications beyond the scope of simulation thanks to agent communication via UDP/IP network protocol with only minor adaptation to the real life building energy system necessary. Moreover, the separation of agent logic and cost functions allows changing of the optimization goal for each individual user without interfering with the agent system and empowers other developers to design own cost functions for their individual optimization goal. To the best of the authors knowledge, no other MAS offers this functionality.

\section{System architecture}

In the following sections the roles of different types of agents, the system architecture, and the communication architecture of the Modelica library will be discussed.

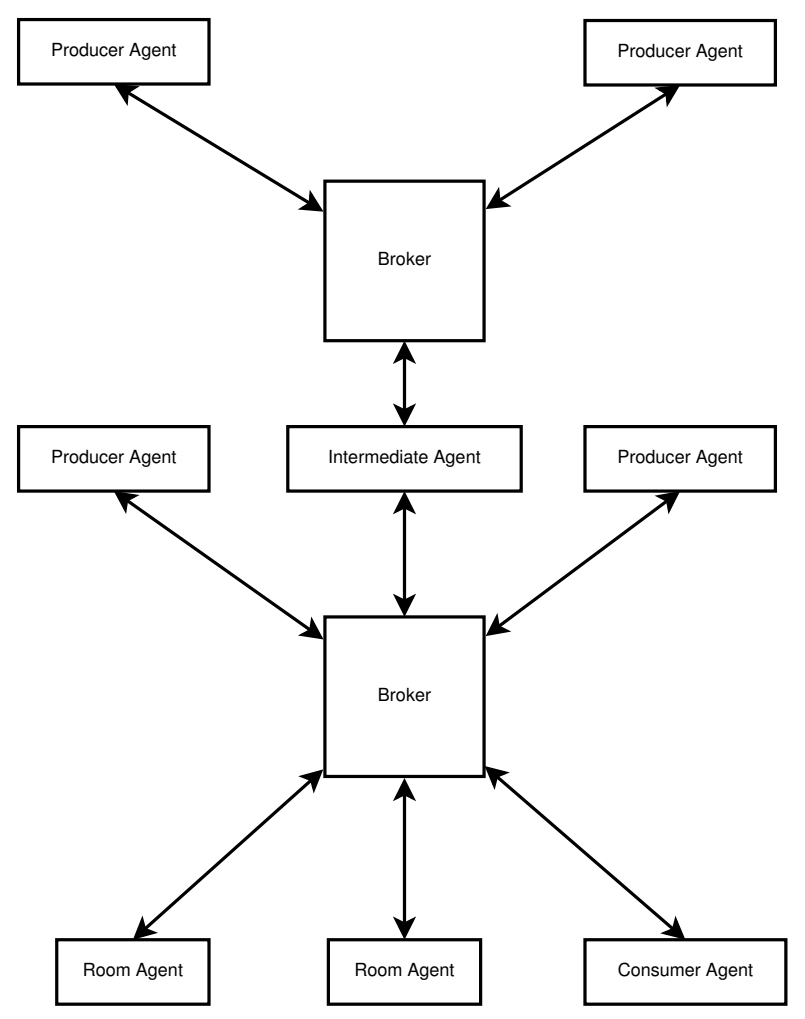

Figure 1. Structure of the Multi-Agent System 

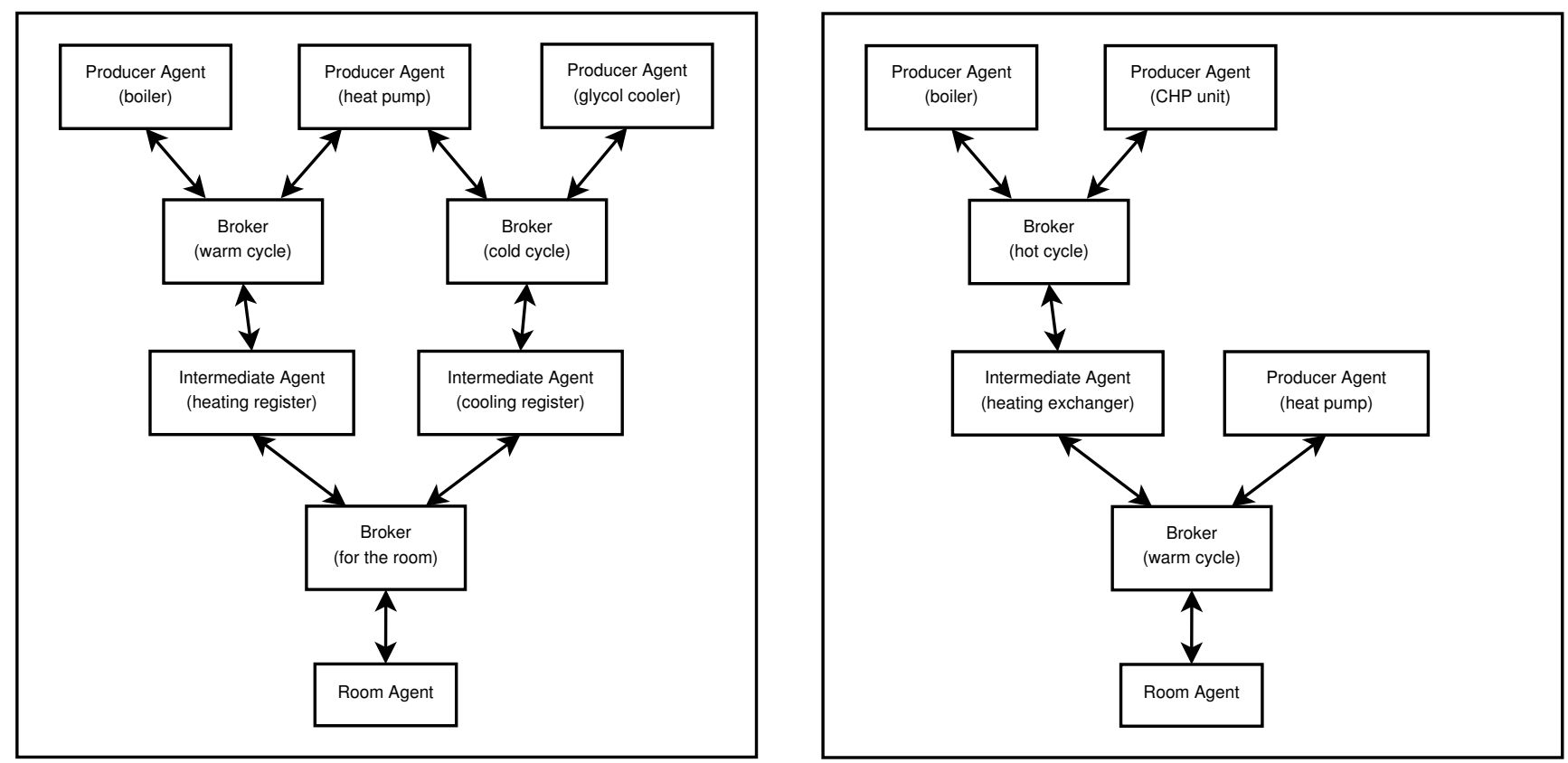

Figure 2. Cascading of the agent system with the help of the intermediate agent

\subsection{Fundamentals}

The agents are designed as state machines, which is a type of event discrete system, meaning that one agent can take on one state and change to a different state when a transition condition becomes true. For the implementation of the state machines, the Modelica StateGraph library (Otter et al., 2005) was used.

The contentual agent communication is based on the FIPA ACL Message Structure Specification (FIPA, 2002a) and FIPA Communicative Act Library Specification (FIPA, 2002b), which depict a common standard for agent communication. Physical agent communication is established via UDP/IP standard under the use of the Modelica DeviceDrivers library (Thiele and Bellmann, 2015). The UDP/IP standard allows communication outside of the simulation environment of Modelica, giving the opportunity to operate agents on different machines and potentially hardware-in-the-loop simulation. As UDP does not guarantee message delivery, measurements to guarantee delivery are implemented on the application level.

\subsection{MAS structure}

Figure 1 shows the structure of the MAS. The system is a partially centralized market-based approach in which capacity adjustments of heat and cold are traded. There are four different types of agents in the system: room/consumer agent, producer agent, intermediate agent and broker.

Room/consumer agents represent entities in the system which require heat or cold for their proper functioning within a building energy system, for example rooms, storage tanks or lab equipment which requires process heat. Their task is to estimate a need for heat or cold and to make a corresponding request to the broker. Room agents use PID controllers to calculate a capacity request from a deviation between the current room temperature and the set point. The set point and an allowed deviation from the set point can be set for each individual room agent in the system, allowing the occupants of the room to adjust the system according to their needs. Consumer agents can use a variety of strategies to determine the capacity request.

Producer agents represent entities in the system which supply heat or cold, for example boilers, CHP units, heat pumps or chillers. Their task is to sell heat or cold to the broker and to adjust capacity when a deal was successfully made. In order to make an offer, they use a cost-function which matches a capacity adjustment with a corresponding price. The cost function is exchangeable, rendering the optimization of the system performance towards different optimization goals possible.

Intermediate agents are a hybrid of producer agents and consumer agents. They act as a consumer in one market and as a producer in another market. They can represent heat exchangers in building energy systems. With the help of the Intermediate agent, cascaded energy systems can be controlled with the MAS system.

The broker is a purely virtual agent and is not connected to any physical entity of the building energy system. It facilitates the trade of heat and cold by collecting requests from room/consumer agents and asking for offers from Producer agents. After collecting all offers, it chooses the most cost effective supplier and requests a capacity adjustment.

\subsection{Trading procedure}

In the following, the working principle of the whole controlling process will be explained. The room agent notices a temperature which is out of a certain range around the set 
temperature and decides to take action. It sends a heating or cooling request to the broker asking for a certain surplus of heat or cold during a specified period of time. The broker waits for requests of other rooms for a certain time to bundle individual requests to one big request. When a specific waiting time for more requests has expired, the broker calls for proposals from each of the producer agents. The producer agents check if they can supply the requested amount of heat or cold and calculate a corresponding price with the help of a cost function. Afterwards, all producer agents send either an offer or a refusal to the broker. Based on these proposals the broker chooses the best offers and calculates a price for each request from the room agents. This information is sent to the room agents which confirm their request. Based on these confirmed requests, the broker then decides which offer is best-suited for the corresponding request and sends out "accept offer" or "reject offer" messages to the producer agents. Producer agents that received "accept offer" messages adjust their capacity accordingly. The communication procedure between the broker and the producer agents follows the FIPA Contract Net Interaction Protocol specification (FIPA, 2002c).

The intermediate agent is important for the flexible use of the agent system. It allows the cascading of the agents and the interconnection of different heating circuits. In the case of Figure 1, the Intermediate agent communicates as a producer to the lower broker and as a consumer to the upper broker. Thereby the two producers in the upper circle can be addressed although they are not part of the same heating circuit. Two examples of the use of the intermediate agent are shown in Figure 2. On the left-hand side of the figure an HVAC system is described in which each room has a heating and a cooling register. The broker mediates between the room agent and the two registers. As the registers need to be supplied with cold or heat themselves, they are each represented by an intermediate agent, that ensures their supply by buying heat or cold from superior markets. On the right-hand side an HVAC system is described in which each room has only one heating device and no cooling device (common European residential building). There are, however, two temperature circuits with different temperature levels. These circuits surround a heat exchanger represented by an intermediate agent. In case of capacity shortage in the warm temperature circuit, the hot temperature circuit can act as a heat producer by transferring heat via the heat exchanger.

\subsection{Cost functions}

The cost functions are used by the producer agents to calculate a corresponding price for a requested capacity adjustment. In the Modelica Library, producer agents and cost functions are separate components, which means that the cost functions are easily exchangeable, depending on the optimization goal of the MAS. In the library cost functions depending on fuel cost, exergy loss and primary exergy loss are available. For demonstration purposes fuel cost functions are used in the following as these depict the simplest form of available cost functions. The functionality of the exergy cost functions has been proven in (Bünning, 2015).

In the case of fuel cost functions, the cost per hour of operation of a heat/cold producing entity is calculated as following:

$$
\frac{C(c a p)}{h}=\frac{p}{\eta} * c a p
$$

In which cap represents the capacity, $\frac{C(c a p)}{h}$ denotes the cost per hour as a function of the capacity, $p$ stands for the price of the fuel per $\mathrm{kWh}$ and $\eta$ for a representative efficiency factor of the device. The costs for a capacity adjustment follows:

$$
\begin{aligned}
\frac{\Delta C}{h}= & \frac{C\left(\text { cap }_{\text {new }}\right)}{h}-\frac{C\left(\text { cap }_{\text {current }}\right)}{h} \\
& =\frac{p}{\eta} *\left(\text { cap }_{\text {new }}-\text { cap }_{\text {current }}\right)
\end{aligned}
$$

With the help of the calculated $\frac{\Delta C}{h}$, producer agents determine the costs of a capacity adjustment and make an offer to the broker.

\section{Modelica HVAC Agent-based Con- trol Library}

In the previous sections the development of communication and logic concepts, agent behaviour and cost functions have been illustrated. These aspects are combined and implemented in the Modelica programming language resulting in the Modelica HVAC Agent Library.

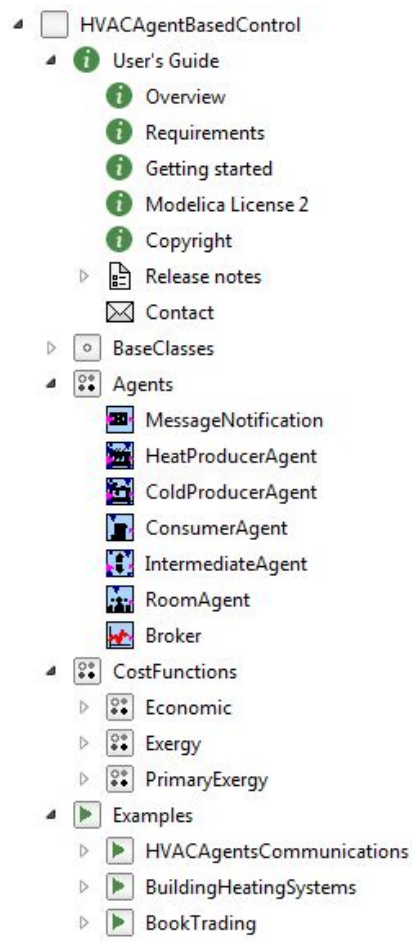

Figure 3. Structure of the HVAC Agent-based Control Library 
Figure 3 shows the structure of the library. It features six different agents for the creation of MAS to control building energy systems. For the producer agents, cost functions with different calculation methods based on fuel costs, exergy and primary exergy are available. The fuel cost functions are applied within this study. The other cost functions have been defined in order to be used in an ongoing research project which aims at developing an exergybased control strategy for building energy systems. Furthermore, the library offers an example agent system with two room agents, one broker and two producer agents, which will be discussed in the following section. Each model of the library is documented regarding its use and function and has its own icon.

Besides the HVAC related agents, agents implementing a book trading example in reference to (Caire, 2009) are included as well as examples to demonstrate agent communication between two different machines.

\section{Case study}

A scheme of the system under investigation is shown in Figure 4. The system consists of two rooms, each equipped with a convective radiator. The rooms are connected to weather data from the 2012 Test Reference Year. Furthermore, the system features two heat supplies, a gas boiler and an electric heating rod. The heating rod can be run on electricity from the grid or on electricity provided by an additional PV panel. The gas boiler has a maximum capacity of $3 \mathrm{~kW}$ and the heating rod of $2 \mathrm{~kW}$.

Both rooms are equipped with a room agent. Both heat sources are equipped with producer agents. To complete the MAS, a broker is used. As cost functions, the fuel cost functions are used with parameters shown in Table 1. Additionally, the cost function of the heating rod is connected to a sensor which measures solar radiation. When solar radiation reaches a value of $310 \frac{\mathrm{W}}{\mathrm{m}^{2}}$, it is assumed that sufficient electricity is provided by the PV panel and therefore considered free of charge. Consequently the fuel price for the heating rod becomes zero.

Table 1. Cost function parameters

\begin{tabular}{lcc}
\hline & $p\left[\frac{\text { Euro }}{k W h}\right]$ & $\eta[-]$ \\
\hline boiler & 0.08 & 0.80 \\
heating rod (grid) & 0.35 & 1.00 \\
heating rod (PV) & 0.00 & 1.00 \\
\hline
\end{tabular}

Besides the control via the agent system, each thermal zone is equipped with a PID controller and a valve, which allow the control of the room temperature to a limited degree.

All physical components of the system are taken from the AixLib library (RWTH-EBC, 2015). The simulation is executed on Dymola 2016 (Dassault Systemes, 2016).

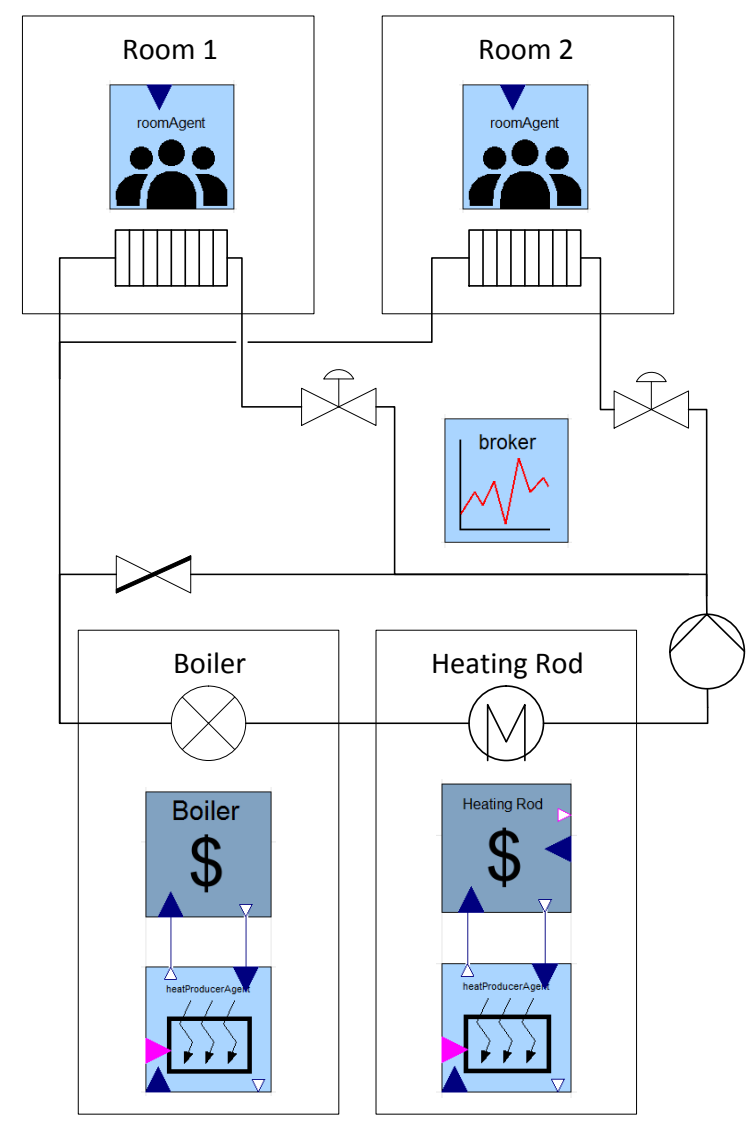

Figure 4. Example heating system with agents

\section{Results and discussion}

The system was simulated for 28 days with the weather data of the month of February of the 2012 Test Reference Year. Figure 5 shows the behaviour of the outside air temperature during the simulated time period. It can be seen that the temperature ranges from $+15^{\circ} \mathrm{C}$ to $-8^{\circ} \mathrm{C}$. The data therefore offers situations of both high and low heating requirement for the simulation.

Figure 6 shows the trend of the air temperature in both rooms. The set point for the room temperature is $20^{\circ} \mathrm{C}$. It can be seen that the temperatures are kept at a satisfactory level between $19.5^{\circ} \mathrm{C}$ and $20.5^{\circ} \mathrm{C}$ during the vast majority of time. Between these temperatures, the control of the room temperature is governed by the PID controllers and valves.

As soon as the room temperature reaches $19.5^{\circ} \mathrm{C}$ or $20.5^{\circ}$, the system leaves the control range of the PID controllers and the room agents become active. In case of $19.5^{\circ} \mathrm{C}$ further heat is requested by the agents, in case of $20.5^{\circ} \mathrm{C}$ a reduction of heat supply is requested. It can be ascertained that the reaction time of the agent system is sufficient as the system rarely crosses the threshold temperatures.

Figure 8 shows the capacities of the boiler and the heating rod, which are solely controlled by the MAS. It can 


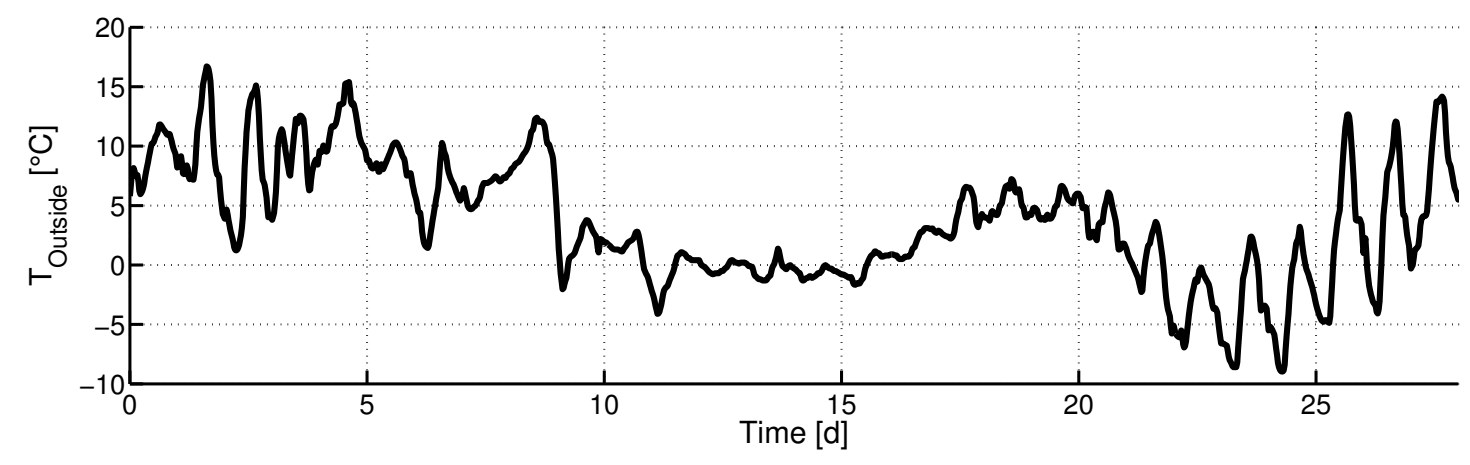

Figure 5. Outside air temperatures

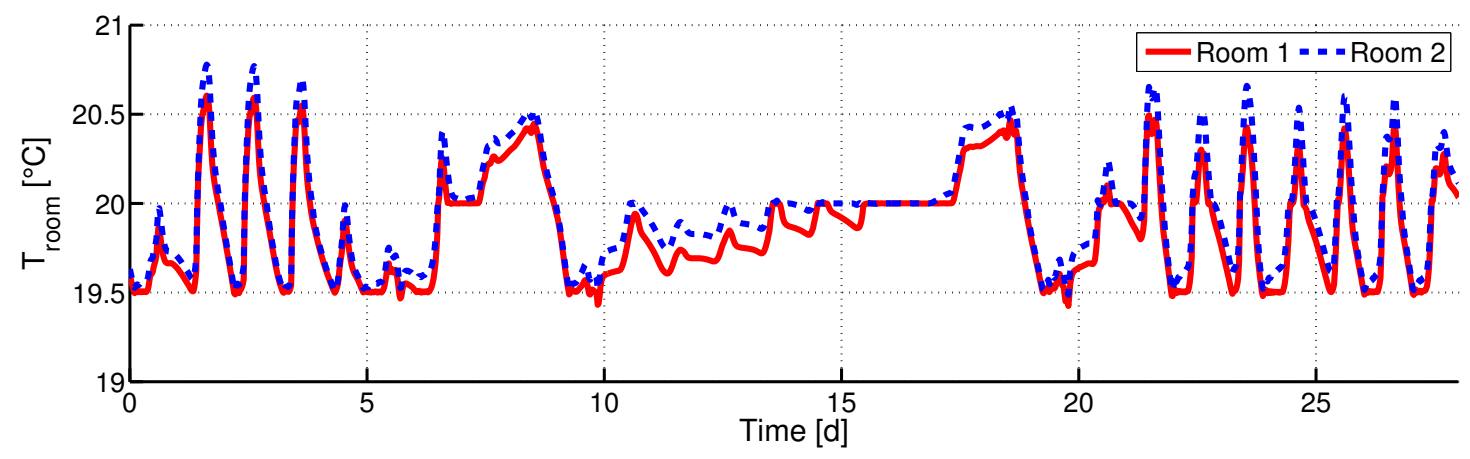

Figure 6. Room temperatures

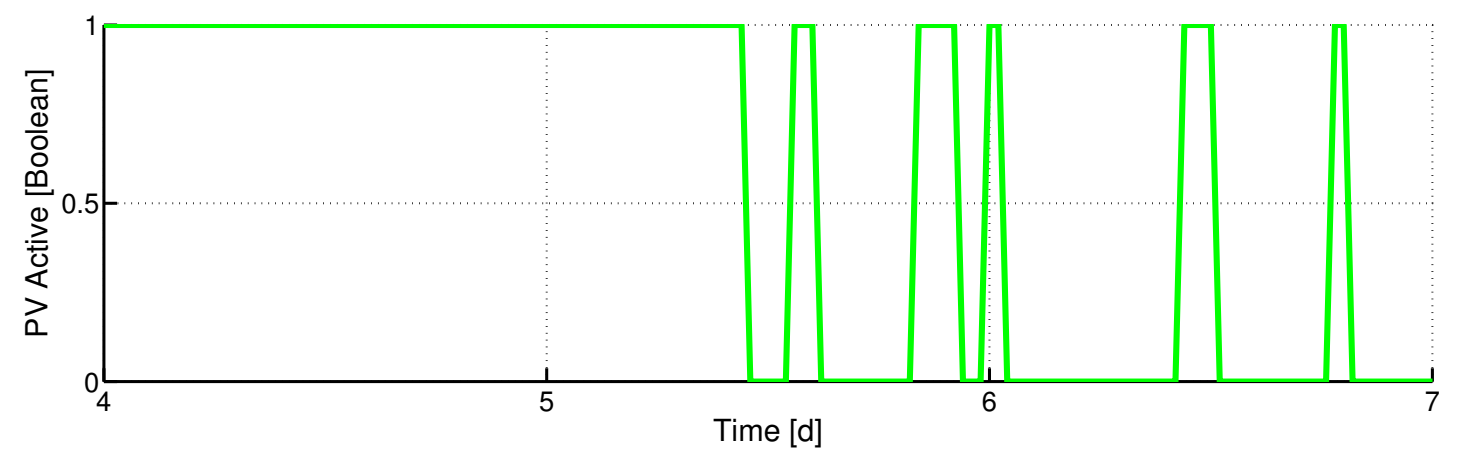

Figure 7. Activeness of PV panel

be seen that the capacities vary based on the current heat demand of the system. The figure also shows that the heat supplies are used less during the first third of the month, when the outside air temperature is generally higher. It can further be seen that the boiler holds the greater share of both heat supplies as it is mostly more cost effective.

Figure 9 shows a detailed segment of the heat supply capacities between day 4 and 7 . Figure 7 shows the corresponding activeness of the PV panel which determines whether the electricity for the heating rod is considered free of charge. A comparison of the figures shows the effect of the different electricity prices on the MAS behaviour. When the heat supplies first become active between day 4 and 5 , it can be seen that the heating rod is only used once the PV panel is active. In the middle of day 5 the heating rod is switched off as the electricity is not free of charge any more. Shortly afterwards, the heat supply is increased by the boiler as the PV is no longer active. When the boiler reaches its maximum capacity in the first third of day six, the heating rod is switched on, although the PV is inactive, as the boiler is not able to supply more heat. It can further be seen that the other active PV times, apart from the long period on days 4 and 5, are not used to the fullest extent as no heat requests are made during these times. Agents used to specifically survey PV activeness could be introduced here.

The functionality of the system was validated in the simulation. However, the MAS can also be used to control real life applications because the agents communicate to each other beyond the border of the simulation software framework as they use a UDP/IP protocol to communicate via an Ethernet network. An example for this functionality is provided in the library. It shows the user how to run an MAS on distributed machines. Such a distributed 


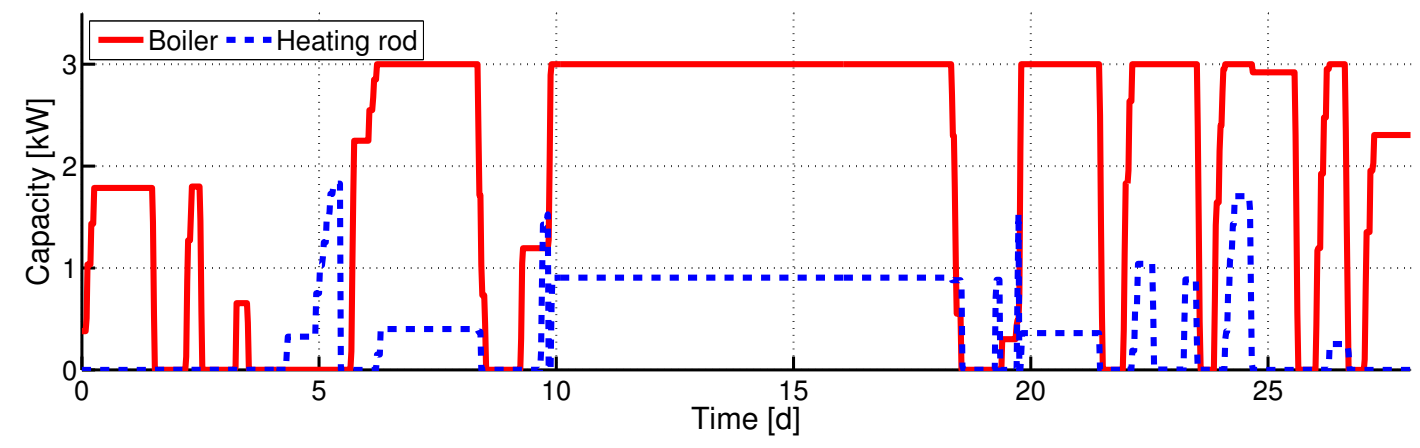

Figure 8. Heat supply capacities

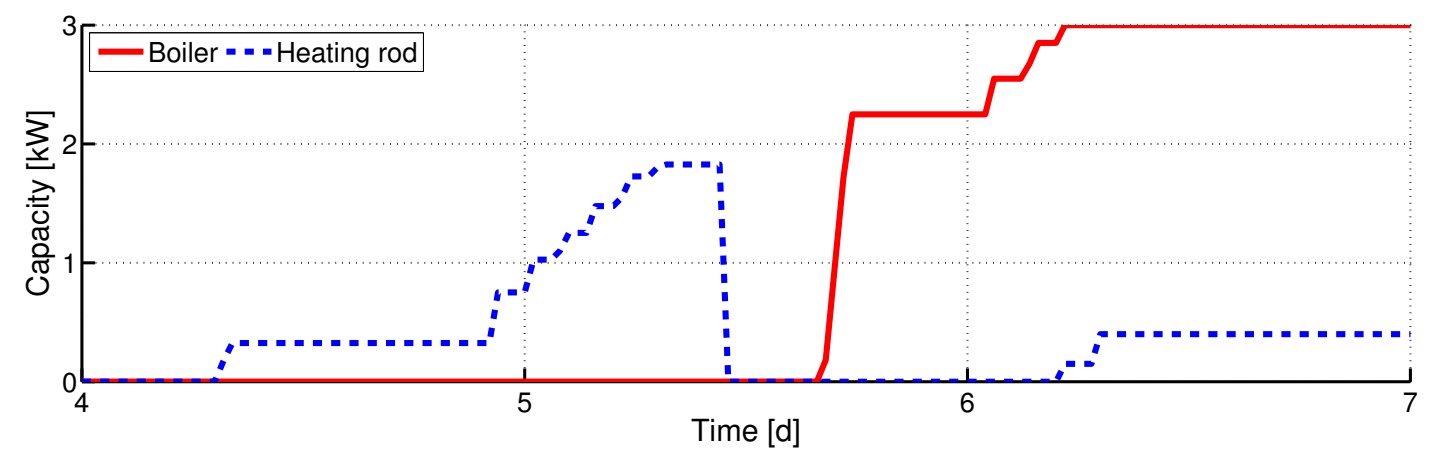

Figure 9. Detailed heat supply capacities

MAS offers much higher reliability compared to a central control as the system is still functional in case an individual producer or consumer agent fails. This means that each entity in the energy system (e.g room, boiler, etc.) is connected to one computer on which the corresponding agent is running. Communication between agent and device needs to be developed individually as each building energy system device has a different software interface. If another entity is added to the system, it can be integrated into the building energy system control with minimal effort by setting it up with its own agent and introducing the agent to the broker. The concept is therefore highly flexible and convenient.

The results show that the MAS is able to maintain a system variable within margin while reducing the effort in accordance to an optimization goal (in this case fuel price). Although the system is developed and tested for building energy simulation, it can be used and extended to optimize any system in which forms of energy are distributed between components, such as chemical processes, district heating systems and electricity smart grids. The interchangeability of the cost function supports this diverse application as any developer can set up own cost functions for their needed domain.

\section{Conclusion}

A Modelica library for the agent based control of building energy systems was introduced. The structure of the Multi-Agent System was explained and the roles of different types of agents were discussed. Furthermore, the trading procedures of capacity adjustments and an example of a type of cost function were introduced. The MAS was tested with an example of a building energy system in the simulation environment of Dymola. The results have shown that the system is capable of keeping a satisfactory room temperature while selecting the heat supply with the most cost effective way of generating heat. The library provides a flexible MAS that can be applied to multiple domains in the energy field due to exchangeable cost functions and that requires minimal implementation effort. Moreover, UDP/IP communication between agents software environment allows real life hardware application in the form of a distributed agent system. Future research will be dedicated to the development of model-predictive cost functions and the application of the MAS to smart district heating grids.

\section{Acknowledgement}

The authors gratefully acknowledge the financial support provided by the BMWi (Federal Ministry for Economic Affairs and Energy), Germany, promotional references 03ET1218A.

\section{References}

Afram, A. and Janabi-Sharifi, F. (2014). Theory and applications of hvac control systems âĂŞ a review of model predictive control (mpc). Building and Environment, 72:343-355.

Ali, M., Vukovic, V., Sahir, M. H., and Fontanella, G. (2013). Energy analysis of chilled water system configurations using simulation-based optimization. Energy and Buildings, 59:111-122. 
Allan, R. (2010). Survey of agent based modelling and simulation tools. Technical report, Science and Technology Facilities Council.

Ansari, J., Gholami, A., and Kazemi, A. (2016). Multi-agent systems for reactive power control in smart grids. International Journal of Electrical Power \& Energy Systems, 83:411-425.

Bellifemine, F. L., Caire, G., and Greenwood, D. (2007). Developing Multi-Agent Systems with JADE. Wiley Series in Agent Technology. Wiley.

Bünning, F. (2015). Development of a modelica-library for the agent-based control of hvac systems. Bachelorthesis, RWTH Aachen University.

Caire, G. (2009). Jade tutorial - jade programming for beginners. TILAB.

Dassault Systemes (2016). Dymola. http://www.3ds.com/productsservices/catia/products/dymola.

Davidsson, P. and Boman, M. (2000). Saving energy and providing value added services in intelligent buildings: A mas approach. In Agent Systems, Mobile Agents, and Applications, pages 166-177. Springer.

Divenyi, D. (2013). Agent-based modeling of distributed generation in power system control. IEEE Transactions on Sustainable Energy, 4:886-889.

FIPA (2002a). Fipa acl message structure specification.

FIPA (2002b). Fipa communicative act library specification.

FIPA (2002c). Fipa contract net interaction protocol specification.

Fuchs, M., Teichmann, J., Lauster, M., Remmen, P., Streblow, R., and Müller, D. (2016). Workflow automation for combined modeling of buildings and district energy systems. Energy.

Huber, M., Brust, S., Schütz, T., Constantin, A., Streblow, R., and Müller, D. (2015). Purely agent based control of building energy supply systems. In ECOS - International Conference on Efficiency, Cost, Optimization, Simulation and Environmental Impact of Energy Systems.

Huberman, B. A. and Clearwater, S. H. (1995). A multi-agent system for controlling building environments. In ICMAS, pages 171-176.

Hurtado, L., Nguyen, P., and Kling, W. (2015). Smart grid and smart building inter-operation using agent-based particle swarm optimization. Sustainable Energy, Grids and Networks, 2:32-40.

Jiang, Z. (2006). Agent-based control framework for distributed energy resources microgrids. In IEEE/WIC/ACM International Conference on Intelligent Agent Technology.

Karavas, C.-S., Kyriakarakos, G., Arvanitis, K. G., and Papadakis, G. (2015). A multi-agent decentralized energy management system based on distributed intelligence for the design and control of autonomous polygeneration microgrids. Energy Conversion and Management, 103:166-179.

Khan, M. R. B., Jidin, R., and Pasupuleti, J. (2016). Multiagent based distributed control architecture for microgrid energy management and optimization. Energy Conversion and Management, 112:288-307.

Kirn, S. (2002). Kooperierende intelligente softwareagenten.
Wirtschaftsinformatik, 44(1):53-63.

Kok, K., Roossien, B., MacDougall, P., van Pruissen, O., Venekamp, G., Kamphuis, R., Laarakkers, J., and Warmer, C. (2012). Dynamic pricing by scalable energy management systemsâĂŤfield experiences and simulation results using powermatcher. In Power and Energy Society General Meeting, 2012 IEEE, pages 1-8. IEEE.

Kuznetsova, E., Li, Y.-F., Ruiz, C., and Zio, E. (2014). An integrated framework of agent-based modelling and robust optimization for microgrid energy management. Applied Energy, 129:70-88.

Mokhtar, M., Liu, X., and Howe, J. (2014). Multi-agent gaussian adaptive resonance theory map for building energy control and thermal comfort management of uclan's westlakes samuel lindow building. Energy and Buildings, 80:504-516.

Mokhtar, M., Stables, M., Liu, X., and Howe, J. (2013). Intelligent multi-agent system for building heat distribution control with combined gas boilers and ground source heat pump. Energy and Buildings, 62:615-626.

Otter, M., Arzen, K., and Dressler, I. (2005). Stategraph âĂȘ a modelica library for hierarchical state machines. In In Proceedings of the 4th International Modelica Conference, pages 569-578.

Perera, D., Winkler, D., and Skeie, N.-O. (2016). Multi-floor building heating models in matlab and modelica environments. Applied Energy, 171:46-57.

Qiao, B., Liu, K., and Guy, C. (2006). A multi-agent system for building control. In Proceedings of the IEEE/WIC/ACM international conference on Intelligent Agent Technology, pages 653-659. IEEE Computer Society.

Radhakrishnan, B. M. and Srinivasan, D. (2016). A multi-agent based distributed energy management scheme for smart grid applications. Energy, 103:192-204.

Rahman, M., Mahmud, M., Oo, A., Pota, H., and Hossain, M. (2016). Agent-based reactive power management of power distribution networks with distributed energy generation. Energy Conversion and Management, 120:120-134.

Rosenschein, J. (1985). Rational Interaction: Cooperation among Intelligent Agents. $\mathrm{PhD}$ thesis, Stanford University.

RWTH-EBC (2015). Aixlib - a modelica model library for building performance simulations. https://github.com/rwthebc/aixlib.

Sangi, R., Baranski, M., Oltmanns, J., Streblow, R., and Müller, D. (2016). Modeling and simulation of the heating circuit of a multi-functional building. Energy and Buildings, 110:13-22.

Sangi, R., Streblow, R., and Müller, D. (2014). Approaches for a fair exergetic comparison of renewable and non-renewable building energy systems. In The 27th international conference on efficiency, cost, optimization, simulation and environmental impact of energy systems. Turku, Finland.

Thiele, B. and Bellmann, T. (2015). Modelica DeviceDrivers. https://github.com/modelica/Modelica_DeviceDrivers.

van Pruissen, O., van der Togt, A., and Werkman, E. (2014). Energy efficiency comparison of a centralized and a multiagent market based heating system in a field test. Energy Procedia, 62:170-179.

Verein Deutscher Ingenieure (2010). Vdi 2653 blatt 1.

Wang, Z., Wang, L., Dounis, A. I., and Yang, R. (2012). Multi- 
agent control system with information fusion based comfort model for smart buildings. Applied Energy, 99:247-254.

Wang, Z., Yang, R., and Wang, L. (2011). Intelligent multiagent control for integrated building and micro-grid systems. In Innovative Smart Grid Technologies (ISGT), 2011 IEEE $P E S$, pages 1-7. IEEE.

Wernstedt, F. (2005). Multi-Agent Systems for Distributed Control of District Heating Systems. PhD thesis, Blekinge Institute of Technology, Department of Systems and Software Engineering.

Wetter, M., Zuo, W., Nouidui, T. S., and Pang, X. (2014). Modelica buildings library. Journal of Building Performance Simulation, 7(4):253-270.

Xydas, E., Marmaras, C., and Cipcigan, L. M. (2016). A multiagent based scheduling algorithm for adaptive electric vehicles charging. Applied Energy, 177:354-365.

Yang, R. and Wang, L. (2013). Development of multi-agent system for building energy and comfort management based on occupant behaviors. Energy and Buildings, 56:1-7.

Ye, D., Zhang, M., and Sutanto, D. (2015). Decentralised dispatch of distributed energy resources in smart grids via multiagent coalition formation. Journal of Parallel and Distributed Computing, 83:30-43. 